Chinese Journal of Organic Chemistry

\title{
硫肽类抗生素类似物合成进展
}

\author{
王守锋 ${ }^{*, a, b}$ 郑庆飞 ${ }^{c}$ 段盼盼 ${ }^{c}$ 刘 文*,c

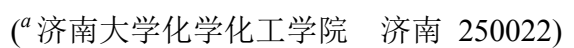 \\ ${ }^{b}$ 山东省氟化学化工材料重点实验室 济南 250022) \\ ( ${ }^{c}$ 中国科学院上海有机化学研究所生命有机化学国家重点实验室 上海 200032)
}

\begin{abstract}
摘要 硫肽类抗生素是一类富含硫元素且被高度修饰的聚噻(噁)唑多肽类天然产物, 该家族化合物以其复杂的分子结 构、良好的生物活性以及新颖的抗菌作用模式而成为研究热点. 近年来, 对于硫肽类抗生素类似物的合成研究发展迅 速. 综述了通过化学半合成、组合生物合成以及前体导向突变生物合成方法获得的硫肽类抗生素类似物的研究进展. 关键词 硫肽类抗生素类似物; 化学半合成; 组合生物合成; 前体导向突变生物合成
\end{abstract}

\section{Progress in Synthesis of Thiopeptide Antibiotics Analogues}

\author{
Wang, Shoufeng*,a,b Zheng, Qingfei ${ }^{c} \quad{\text { Duan, } \text { Panpan }^{c} \quad \text { Liu, Wen }}^{*, c}$ \\ ( ${ }^{a}$ College of Chemistry and Chemical Engineering, University of Jinan, Jinan 250022) \\ ( $b$ Shandong Provincial Key Laboratory of Fluorine Chemistry and Chemical Materials, University of Jinan, Jinan 250022) \\ ( ${ }^{c}$ State Key Laboratory of Bioorganic and Natural Products Chemistry, Shanghai Institute of Organic Chemistry, \\ Chinese Academy of Science, Shanghai 200032)
}

\begin{abstract}
Thiopeptide antibiotics, which are a growing class of sulfur-rich and highly modified polyazolyl peptide natural products, have been appreciated because of their complex structures, potent biological activities and unusual modes of action. Recently, a great deal of effort has been devoted to the development of various approaches for the efficient synthesis of thiopeptide antibiotics analogues. This review summarizes synthetic approaches towards thiopeptide antibiotics analogues via semisynthesis, combinatorial biosynthesis and precursor-directed mutasynthesis.
\end{abstract}

Keywords thiopeptide antibiotics analogue; semisynthesis; combinatorial biosynthesis; precursor-directed mutasynthesis

硫肽类抗生素是一类由微生物次级代谢产生的环 肽类天然产物 ${ }^{[1]}$. 自 1948 年第一例硫肽类抗生素 micrococcin P 被发现以来, 越来越多的硫肽类抗生素成 员被报道, 到目前为止该家族已包含超过一百个成员 ${ }^{[2]}$. 该家族化合物结构复杂, 富含多个噻唑(噁唑)或噻唑啉 (噁唑啉)、多个脱水氨基酸及该家族化合物特有的氮六 元杂环. 根据其核心氮杂六元环取代方式及氧化程度的 差异, Hensens 等 ${ }^{[3]}$ 首次将硫肽类抗生素分为 $\mathrm{a}, \mathrm{b}, \mathrm{c}, \mathrm{d}, \mathrm{e}$ 五个亚家族. a: 哌啶类, 如 $s c h 18640 ; b$ : 脱氢哌啶类, 如 thiostreptons; c: 二氢咪唑哌啶类, 如 sch40832; d: 三 取代吡啶类, 如 micrococcins 和 thiocillins; e: 差弪化吡 啶类, 如 nosiheptide(图 1).
硫肽类抗生素大多具有良好的抗革兰氏阳性菌生 理活性, 尤其是对多种耐药致病菌如耐新青霉素金黄色 葡萄球菌(MRSA), 耐青需素肺炎链球菌(PRSP) 和多耐 药性粪肠球菌(MREF)等具有很好的抑制作用. 研究发 现，硫肽类抗生素是以核糖体为靶点抑制蛋白质的合成 从而达到抑菌的效果 ${ }^{[4 \sim 6]}$, 但其作用模式与目前临床上 应用的抗生素均不同, 因此具有很大的成药潜力. 尽管 硫肽类抗生素具有良好的抗菌活性，但是其水溶性差、 生物利用度低, 从而限制了它们在临床上的应用. 为了 克服硫肽类抗生素成药性方面的不足, 人们尝试用各种 化学合成和生物合成方法制备硫肽类抗生素类似物, 以 便从中篮选活性好、水溶性好的潜在成药分子.

*Corresponding authors. E-mail: chm_wangsf@ujn.edu.cn; wliu@sioc.ac.cn

Received February 15, 2017; revised March 20, 2017; published online April 10, 2017

Project supported by the National Natural Science Foundation of China (No. 31430005) and the Natural Science Foundation of Shandong Province (No. ZR2016HM44).

国家自然科学基金(No. 31430005)、山东省自然科学基金(No. ZR2016HM44)资助项目. 


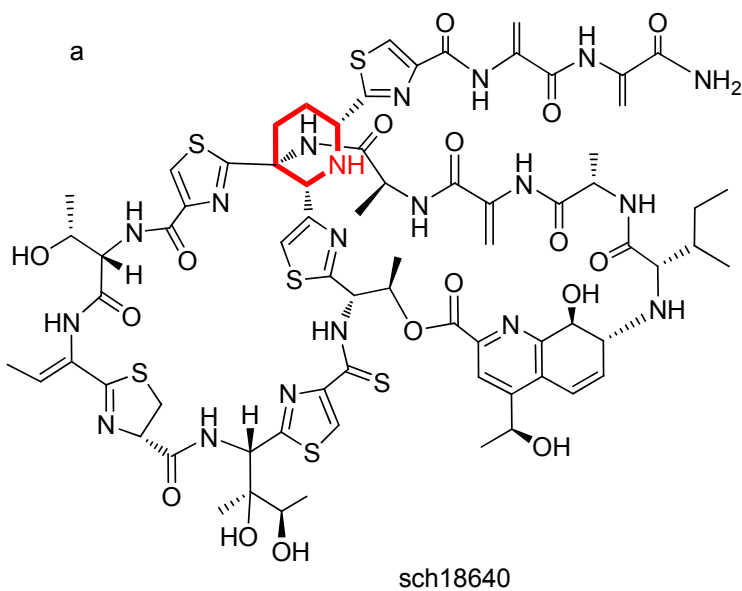

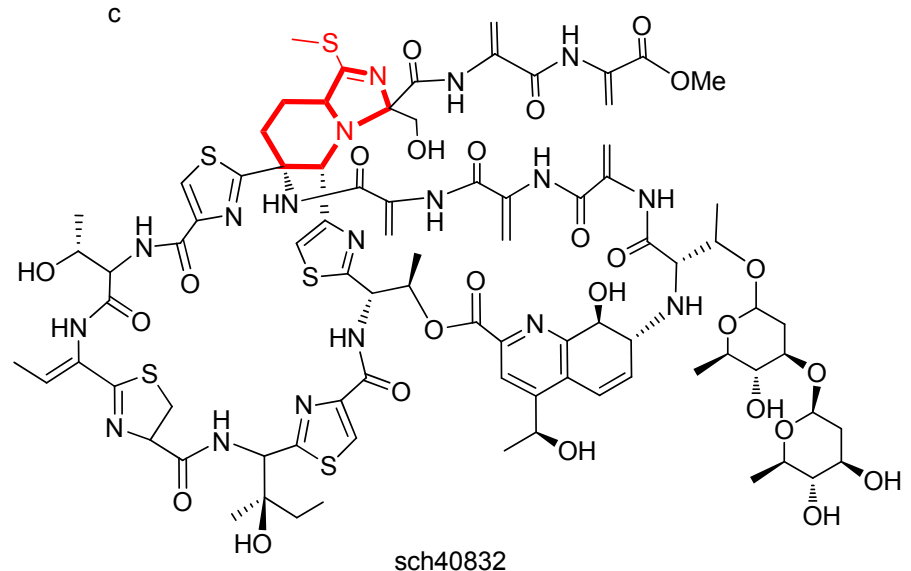

d

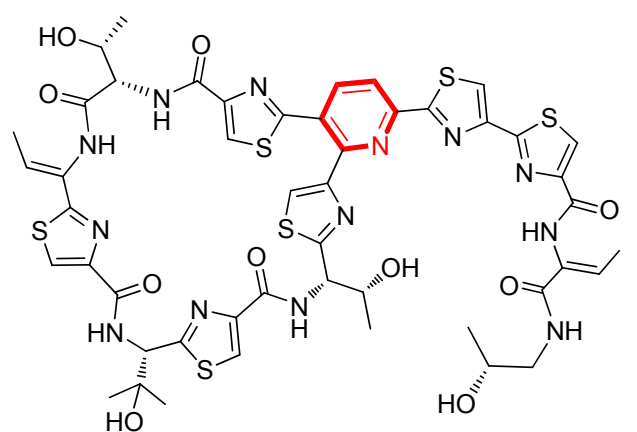

thiocillin 1

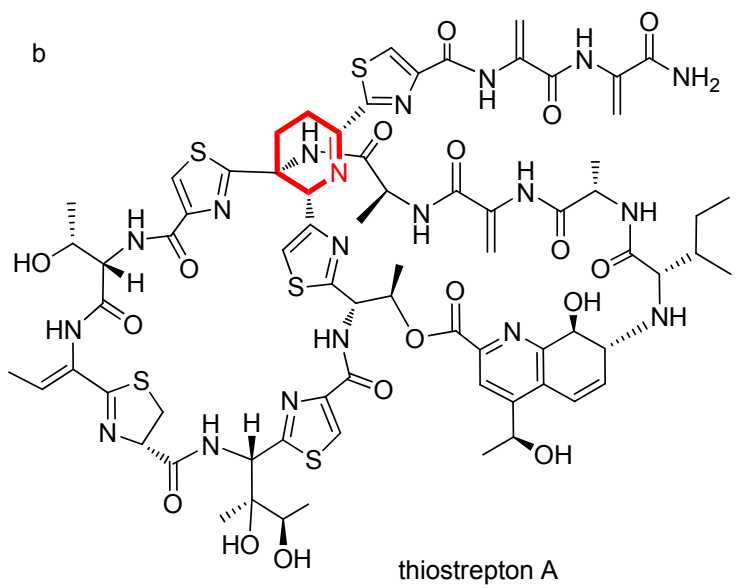

d

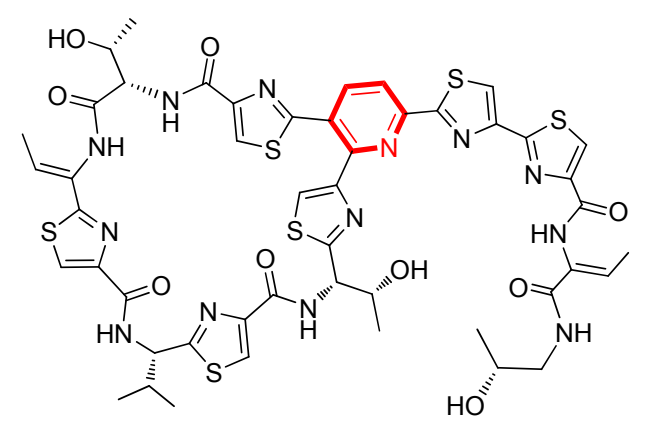

micrococcin $\mathrm{P}$

nosiheptide

图 1 硫肽类抗生素的结构及分类

Figure 1 Structure and classification of thiopeptide antibiotics

\section{1 化学半合成}

通过对硫肽类抗生素分子的局部基团进行化学修 饰获得其类似物是硫肽类抗生素类似物化学半合成中 普遍使用的方法. 硫肽类抗生物分子结构中存在的活泼 反应位点和官能团可以作为发展其类似物的切入点. 化 学半合成方法在 nocathiacin I, GE2270A 及 thiostrepton $\mathrm{A}$ 类似物的合成中取得了丰硕的成果.

\subsection{Nocathiacin I 类似物的化学半合成}

Nocathiacin I 是一个高度后修饰的硫肽类抗生素, 其可发生化学修饰的位点如图 2.

目前，对该分子的化学修饰主要集中在空间位阻小 的分子末端. 最常用的修饰是亲核试剂对末端脱水丙氨 酸(dehydroalanine, Dha)的 Michael 加成 ${ }^{[7]} .2005$ 年, Naidu 小组 ${ }^{[8]}$ 通过这种方法得到了胺基修饰化合物 6 和 


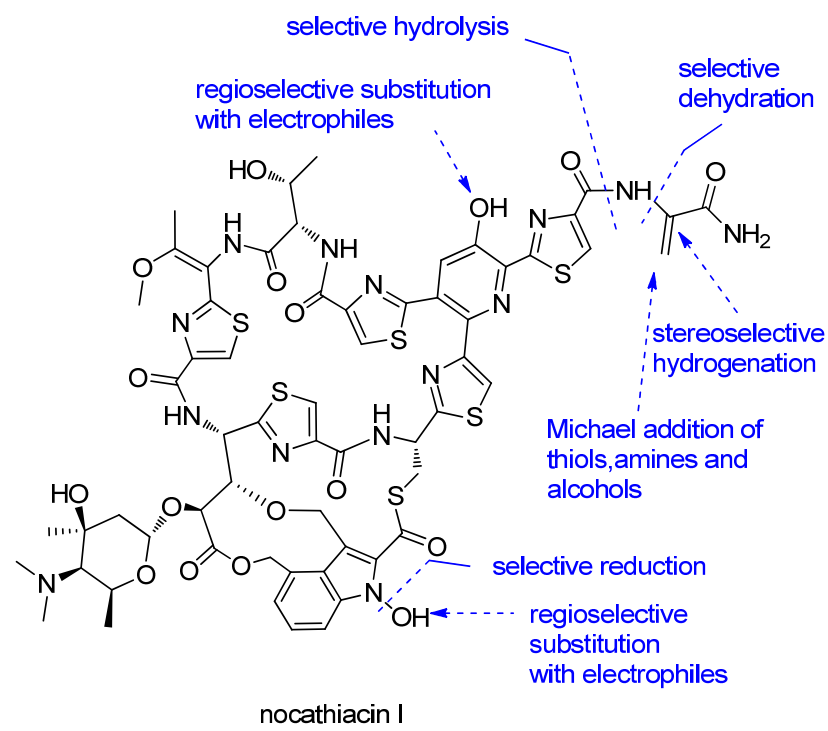

图2 Nocathiacin I的化学半合成修饰

Figure 2 Semisynthetic modifications of nocathiacin I

含硫醚化合物 7, 化合物 6 可以进一步被修饰得到含尿 素基团的化合物 8, 而化合物 7 可以进一步转化为含砜 基的化合物 9. 2006 年, Naidu 小组 ${ }^{[9}$ 将末端双键立体专 一性地还原获得化合物 $\mathbf{1 0}$, 化合物 $\mathbf{1 0}$ 可以进一步脱氨
得末端羒酸化合物 11 , 在化合物 11 的基础上又可以引 入水溶性基团得到化合物 $\mathbf{1 2}$ (Scheme 1). 化合物 8 和 9 的抗菌活性相比于 nocathiacin I 都有所降低, 降低为 nocathiacin I 的十分之一. 化合物 $\mathbf{1 2}$ 在抗菌活性保持的 情况下水溶性提高了近 10 倍(表 1).

此外, nocathiacin I 的末端还可以选择性地降解获 得两种类型的产物: 一种是诺卡噻唑菌酸(nocathiacin acid), 另一种是相对应的末端酰胺化的诺卡噻唑菌素 (nocathiacin IV) $)^{[10,11]} .2007$ 年, $\mathrm{Liu}$ 小组 ${ }^{[12,13]}$ 报道了诺卡 噻唑菌酸可以通过与含有胺基基团的化合物缩合获得 进一步修饰的类似物. 2002 年, Ueda 小组 ${ }^{[14]}$ 报道了诺卡 噻唑菌素 IV 可以通过一锅法 $N$-烷基化/还原胺化引入长 的烷基链, 如化合物 13 (Scheme 1). 这些末端修饰获得 了一些水溶性改善且具有良好生物活性的类似物, 有些 对万古霉素抗性的致病菌有较好的活性(表 1$)^{[15]}$.

在对其环骨架修饰方面, Naidu 等 ${ }^{[16,17]}$ 报道了两例大 环上 2 -羊基基吡啶和侧环上 $N$-羊基吲哚的衍生, 其中有膦 酰氧基甲基修饰的化合物 $\mathbf{1 4}$ 和 $\mathbf{1 5}$ (Eq. 1)在提高了水溶 性的同时都保持了良好的体内体外活性 ${ }^{[16]}$. 由于膦酰氧 基甲基醚能够被磷酸酶催化水解并释放 nocathiacin I, 因 此这两种化合物具有成为 nocathiacin I 前药的潜力 ${ }^{[18]}$.

A

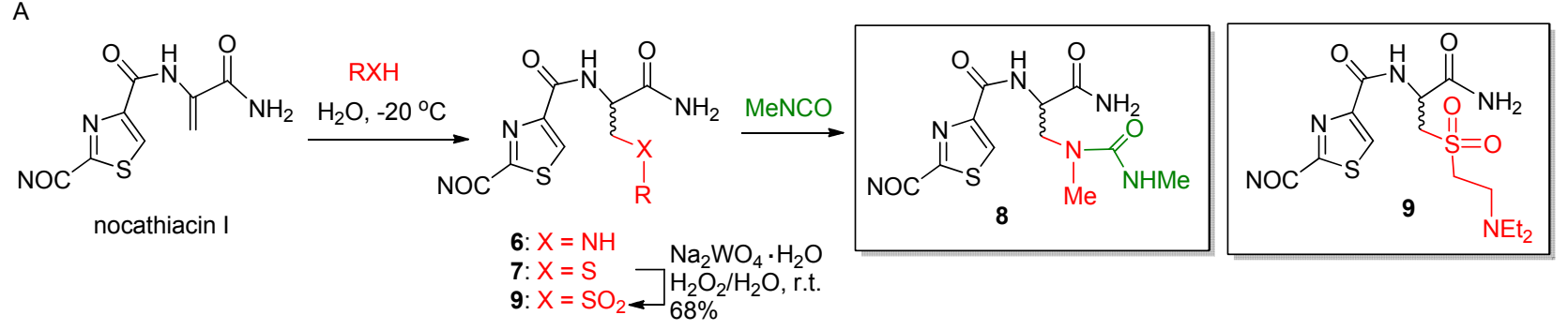

B

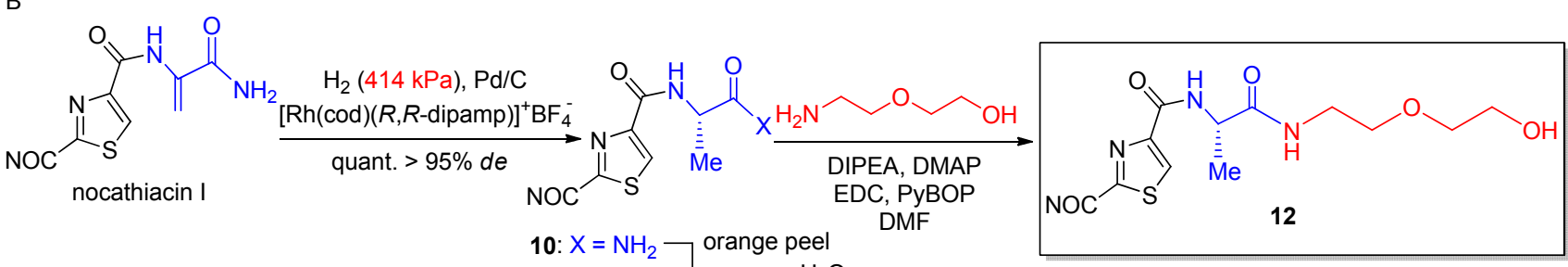
C $\quad 88 \% \mid \begin{aligned} & \text { Mel-HI, THF } \\ & 45^{\circ} \mathrm{C}\end{aligned}$<smiles>N#Cc1nc(C(N)=O)cs1</smiles>
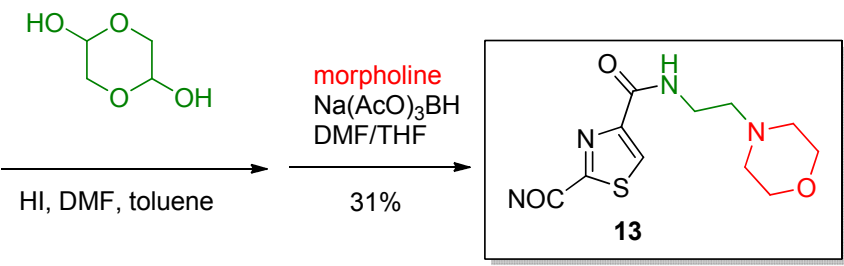

图式 1 Nocathiacin I 尾巴部分的化学半合成修饰

Scheme 1 Semisynthetic modifications of nocathiacin I tails 
表 1 Nocathiacin I 类似物的抑菌活性 ${ }^{a}$

Table 1 Inhibitory activity of nocathiacin I analogues

\begin{tabular}{|c|c|c|c|c|c|c|c|c|}
\hline 化合物 & Nocathiacin I & Nocathiacin IV & 8 & 9 & 13 & 12 & 14 & 15 \\
\hline $\mathrm{MIC}^{a} /\left(\mu \mathrm{g} \bullet \mathrm{mL}^{-1}\right)$ & 0.007 & 0.03 & 0.06 & 0.06 & 0.015 & ND & ND & ND \\
\hline $\mathrm{PD}^{2} 0^{b} /\left(\mathrm{mg} \bullet \mathrm{kg}^{-1}\right)$ & 0.8 & $\mathrm{ND}^{b}$ & ND & ND & ND & 1 & 3 & 0.15 \\
\hline
\end{tabular}

${ }^{a}$ PD50 : dose required to cure $50 \%$ of the animals infected; MIC: minimum inhibitory concentration; ${ }^{b}$ ND: not test.

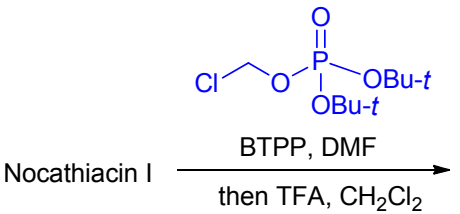

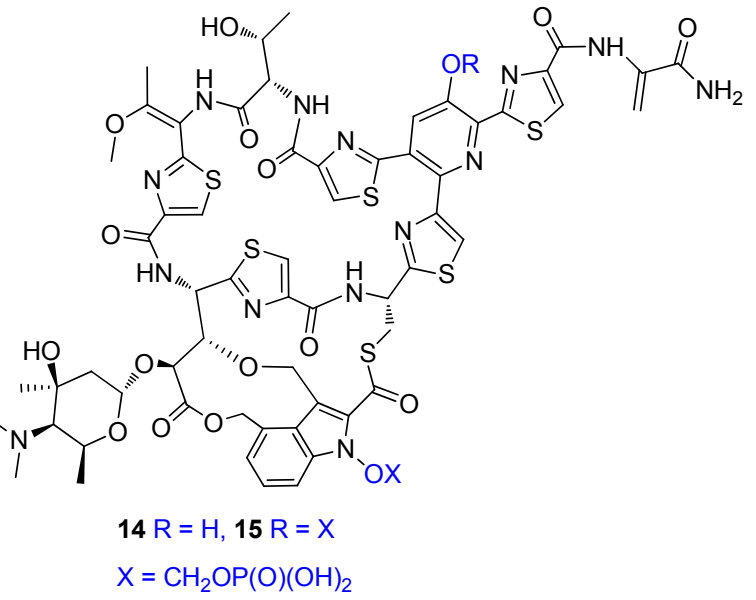

\subsection{GE2270A 类似物的化学半合成}

GE2270A(图 3)的结构中不包含有脱水氨基酸残基, 因此没有 Michael 加成的受体, 尽管如此, 该分子却是 硫肽类抗生素成员中化学半合成研究最为详尽的硫肽 分子之一 ${ }^{[19]}$, 这主要归结于该分子结构具有特殊的末 端结构. 其另外一个反应位点是大环骨架上的苯丝氨酸 侧链 ${ }^{[20,21]}$, 该结构单元可以被修饰甚至被转变成甘氨酸 残基. 由于对大环的修饰不能获得令人感兴趣的分子, 因此大部分对 GE2270A 的化学半合成工作集中在末端 尾巴的修饰.

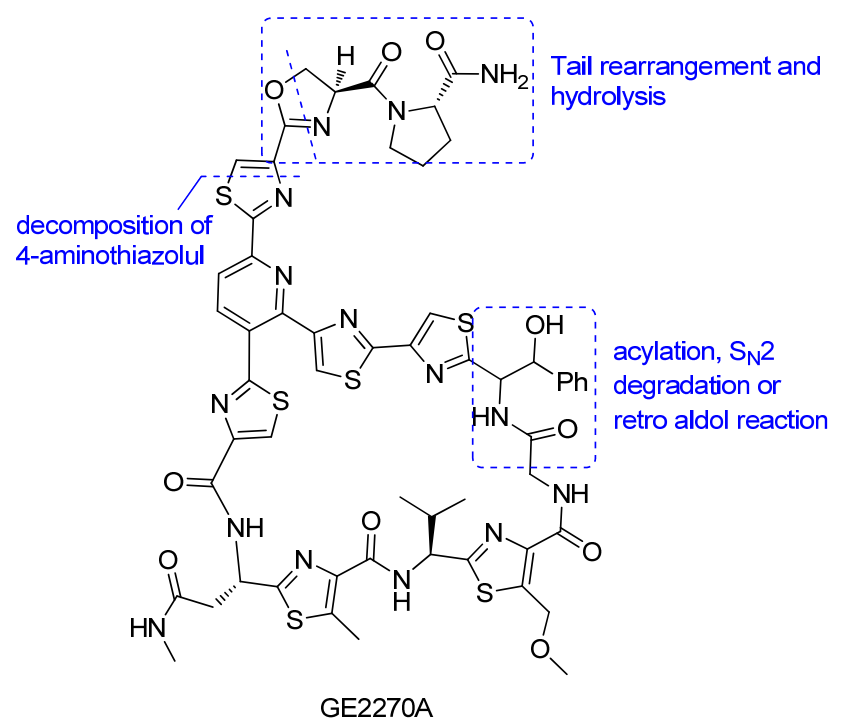

图 3 GE2270A 的化学半合成修饰

Figure 3 Semisynthetic modifications of GE2270A
合成化学家对 GE2270A 末端的修饰主要是降解, 降解后的产物可以进一步发生官能团修饰(Scheme 2). 对 GE2270A 的酸处理可以获得末端重排化合物 17, 化 合物 17 在碱性条件下可以水解形成化合物 18 或者被 $\mathrm{NaBH}_{4}$ 还原为化合物 19. 化合物 18 可以与胺或者肽发 生缩合，化合物 19 也可与羧酸发生缩合反应. 通过这种 方法人们获得了大量的类似物, 但是仅有少数保留了活 性并具有好的水溶性.

2011 年以来, LaMarche 等 ${ }^{[22 ~ 24]}$ 对 GE2270A 末端的 结构改造工作, 取得了显著的成果. 通过 Curtius 重排, 他们将降解掉末端尾巴的 18 转化为 4-胺基噻唑类似物. 他们对 4-胺基噻唑类似物的胺基进一步修饰, 得到的吡 啶酰胺及咪唑类似物活性都不理想 ${ }^{[25]}$, 而从酰胺或胺 基甲酸酯修饰的 4-胺基噻唑类似物中篎选出的环己醇 酸系列类似物, 如化合物 20 , 则表现出很好的活性 ${ }^{[22]}$. 2012 年，该小组在化合物 $\mathbf{2 0}$ 的基础上做了进一步修饰, 在胺基甲酸酯的胺基上引入末端含有羧基的烷基链以 进一步提高水溶性. 在此基础上获得的 GE2270A 类似 物 LEF571(化合物 21) 是第一例用于临床试验的硫肽类 化合物，它能有效地抑制由艰难梭状芽 (Clostridium difficile)引起的肠道感染 ${ }^{[24]}$. 由于其渗透膜能力差, 因 此口服 LEF571 主要是通过粪便排出, 血液中浓度很低. 正是由于这一特性, 使得 LEF571 成为治疗由 Clostridium difficile 引起的肠道感染的潜力药 ${ }^{[26,27]}$, 目 前 LEF571 已经完成了临床 II 期试验.

2015 年, Fabbretti 小组 ${ }^{[28]}$ 对 GE2270A 的末端修饰获 得 NAI003 (22), 该化合物的修饰没有引入距噻唑至少 
A

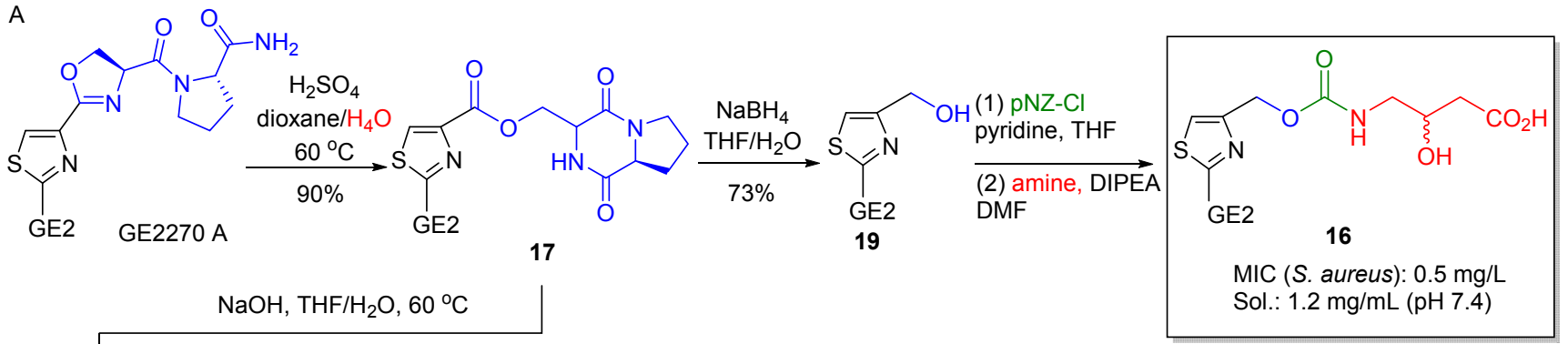<smiles>[R]OC(=O)c1csc(C(=O)O)n1</smiles>

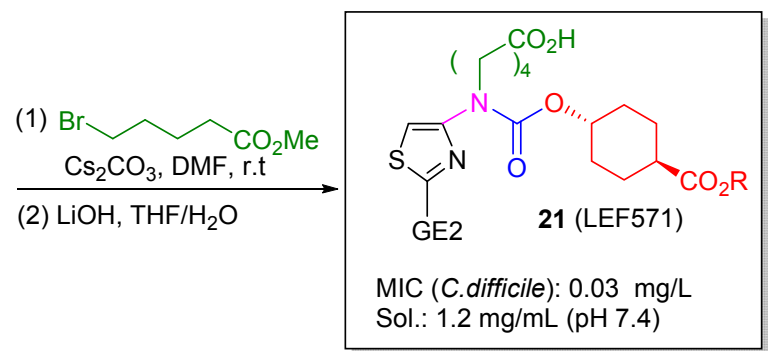

$31 \%$ ( 2 steps)

图式 2 GE2270A 尾巴部分的化学半合成修饰

Scheme 2 Semisynthetic modifications of GE2270A tails

五个原子的羧酸模体. 根据先前其他小组对化学半合成 的 GE2270A 类似物的 SAR 研究, NAI003 的抗菌活性相 比于其母体 GE2270A 不会有大的提高. 生物活性测试 发现其抗菌谱相比于其母体化合物明显缩小, 仅对几个 革兰氏阳性菌有活性且抗菌活性不如其母体 GE2270A. 对 NAI003 敏感的细菌包括痤疮丙酸杆菌(Propionibacterium acnes, P. acnes)家族中的 21 种测试菌. 虽然 NAI003 抗痤疮丙酸杆菌的活性不如 GE2270A, 但其窄 的抗菌谱使得它在对痤疮丙酸杆菌发挥作用的同时不 致杀死皮肤表面有利的共生菌. 这一优点使 NAI003 具 有成为临床上治疗痤疮新药物的潜力, 目前 NAI003 已 经完成了临床 I 期试验.

\subsection{Thiostrepton A 类似物的化学半合成}

Thiostrepton A 的分子结构更为复杂, 其结构中可 修饰的位点(尤其是大环和侧环结构中)比 nocathiacin I 和 GE2270A 更多. 另外, thiostrepton A 除了具有前两种 化合物具有的抗菌活性外, 还具有抗肿瘤和抗㾏活性, 因此也被一些合成化学家选作母体化合物进行化学修 饰, 其可化学修饰的位点如图 4. 这些获得的 thiostrepton A 类似物主要用于研究其抗菌的构效关系 (SAR)及其作用机制、抗肿瘤的 SAR 及其作用机制.

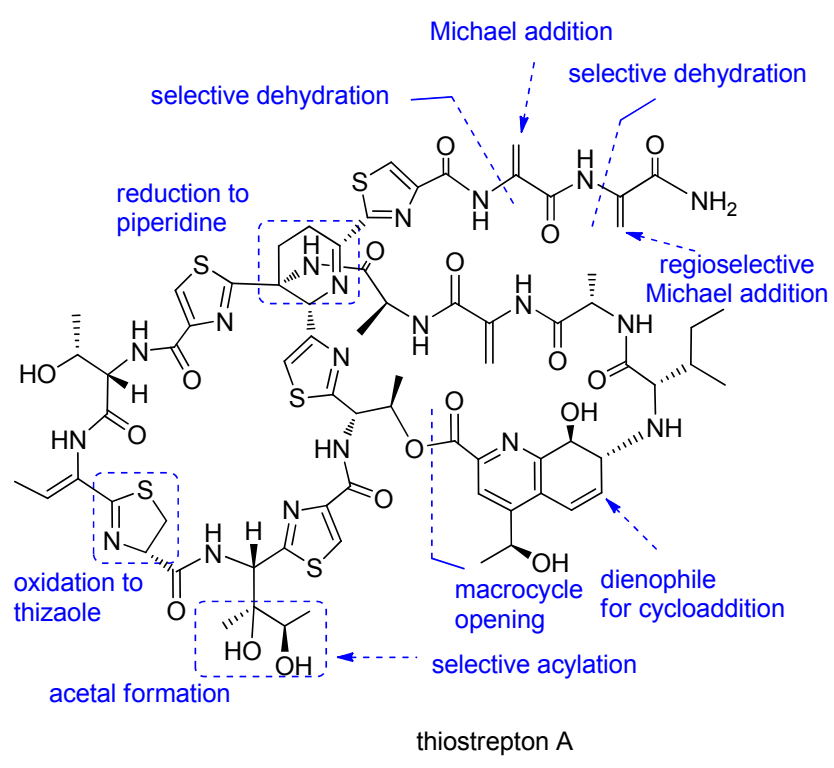

图 4 Thiostrepton A 的化学半合成修饰

Figure 4 Semisynthetic modifications of thiostrepton A

在对 thiostrepton A 的抗菌的 SAR 和作用机制研究 方面, 人们主要集中对其末端尾巴的修饰(双键的 Michael 加成或末端尾巴的降解). 2009 年, Arndt 小组 ${ }^{[29]}$ 通过 Michael 加成, 在截短的化合物 25 的末端双键上引 
入苂光基团获得化合物 $\mathbf{2 8}$, 苂光标签的引入使得他们 发现双环类硫肽 thiostrepton A 在与 $23 \mathrm{~S}$ rRNA/L11 蛋白 相互作用的过程中, 23S rRNA 上 A1067 和 A1095 对形 成复合体起着至关重要的作用 ${ }^{[30]} .2010$ 年, Honek 小 组 ${ }^{[31}$ 通过 Michael 加成到 thiostrepton A 的末端尾巴引入 硫酸基团获得化合物 $\mathbf{3 0}$, 化合物 $\mathbf{3 0}$ 在体外能很好地抑 制革兰氏阴性菌蛋白合成, 但是却对革兰氏阴性菌无抗 菌活性. 这一结果证实硫肽分子对抗革兰氏阴性菌无体 内抗菌活性是由于其无法穿透革兰氏阴性菌的细胞壁. 在对大环骨架修饰方面, Jonker 等 ${ }^{[32]}$ 选择性地将硫链丝 菌素(TSR)核心脱氢哌啶还原可以获得化合物 29, 模拟

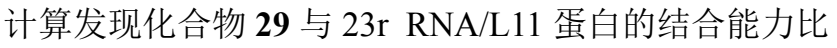
thiostrepton A 更强, 这预示着天然 thiostrepton A 可能并
不是其靶点的最适底物(Scheme 3).

在对 thiostrepton A 抗肿瘤的 SAR 和机制研究方面, 人们对 thiostrepton A 结构上的多个可修饰位点进行了 尝试(Scheme 3). 在大环骨架修饰方面: 2011 年, Balasubramanian 小组 ${ }^{[33]}$ 利用异亮氨酸残基上的双差基合成 了具有缩醛结构的化合物 22, 但是该结构不稳定, 无法 分离. 在侧环修饰方面: 对喹菜啶酸(quinaldic acid, QA) 上的双键环加成获得化合物 23 , 但化合物 23 的抗肿瘤 机制活性完全消失. 在末端尾巴修饰方面: 末端降解掉 两个脱氢丙氨酸的化合物 24 , 降解掉一个脱氢丙氨酸 的化合物 25 及在其末端双键引入生物素获得的化合物 27, 不再具有抗肿瘤活性或抗肿瘤活性明显降低. 在末 端倒数第二个脱氢丙氨酸双键上引入生物素获得的

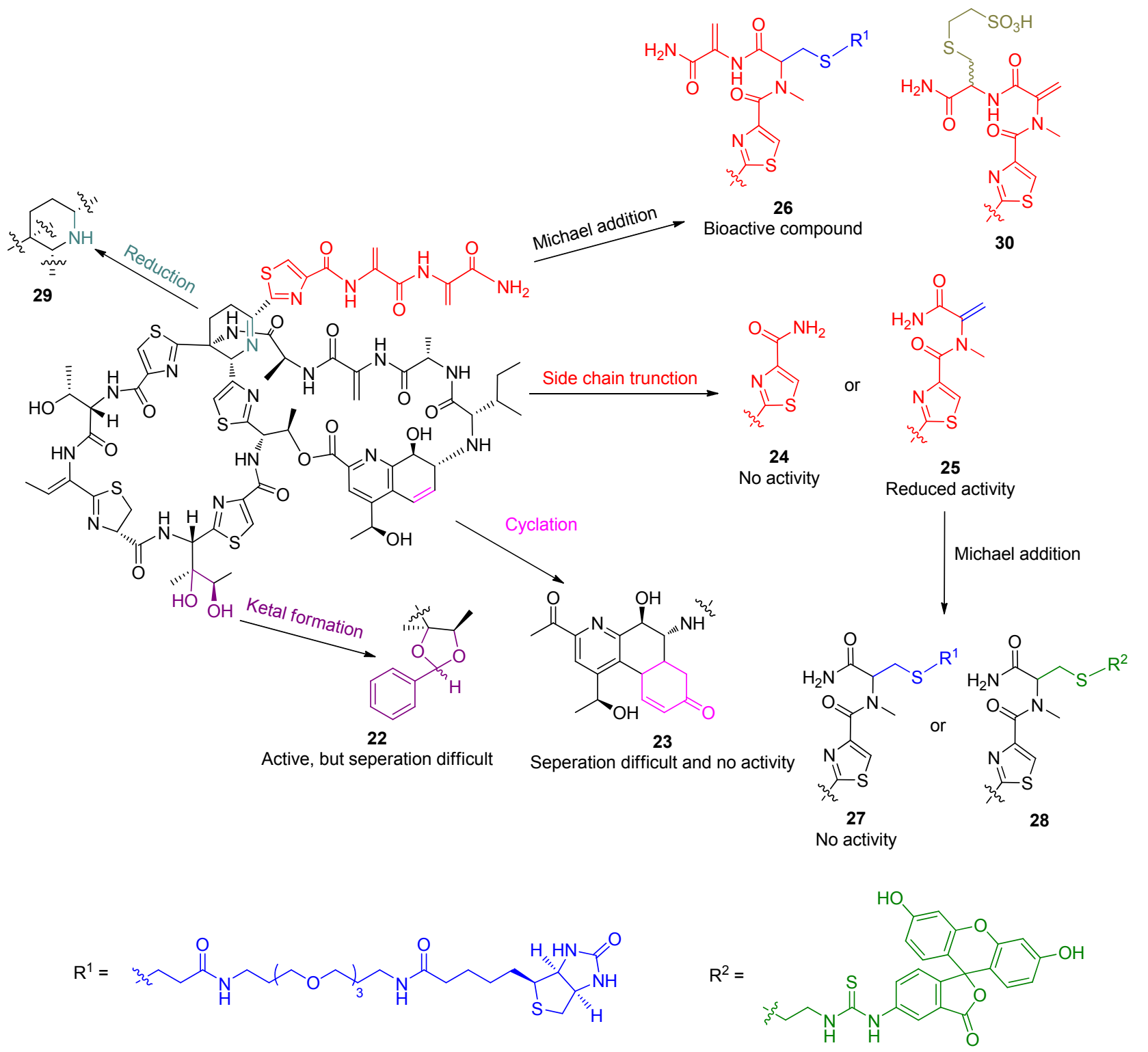

图式 3 Thiostrepton 尾巴和环的半合成修饰

Scheme 3 Semisynthetic modifications of thiostrepton A tails and macrocycles 
化合物 26 保持了抗肿瘤活性. 以带有生物素探针的化 合物 26 对其抗肿瘤机制研究发现: thiostrepton A 是通过 直接与 FOXM1 结合, 从而抑制 FOXM1 与基因组作用 位点结合, 进而降低 FOXM1 下游基因的表达.

\section{2 组合生物合成}

近年来, 对硫肽类抗生素的生物合成研究为获取硫 肽类似物提供了新的思路. 自 2009 年硫肽生物合成基 因族被报道以来, 硫肽类抗生素的生物合成途径是核糖 体的来源已经被多个研究组阐明. 核糖体来源途径意味 着在硫肽抗生素的生物合成中, 前体肽的合成遵循中心 法则, 即分子结构中氨基酸序列是由基因编码的. 这意 味着可以通过引入非天然氨基酸或者前体肽氨基酸的
定点突变来获取硫肽类抗生物的类似物. 另外，许多硫 肽类抗生素成员的前体肽基因和其后修饰基因位于同 一个生物合成基因簇中，这意味着可以通过基因敲除的 手段获得其类似物.

\section{1 非天然氨基酸的引入获取类似物}

2016 年, Young 小组 ${ }^{[34]}$ 报道了在 thiocillin 体系中引 入非天然氨基酸的工作(图 5). 他们把正交琥珀抑制基 因(pyrrolysyl-tRNA 合成酶/tRNApylCUA)引入到 thiocillin 产生菌 B. cereusz 中, 并分别将 3 位、4 位、6 位、 8 位和 13 位氨基酸对应的密码子突变成为终止密码 子 TAG，在发酵体系中加入 BocK, AlocK 或 ProcK, 最 终获得 45 种引入非天然氨基酸的 thiocillin 类似物. 引
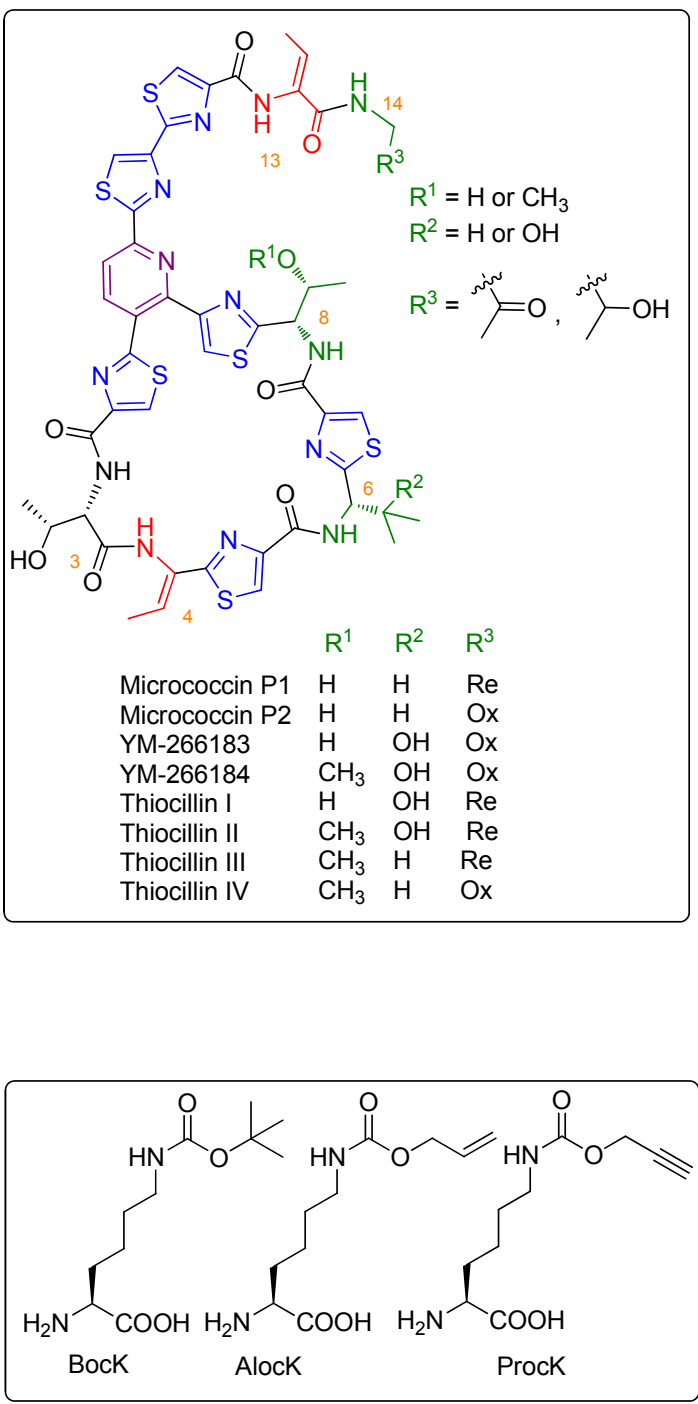
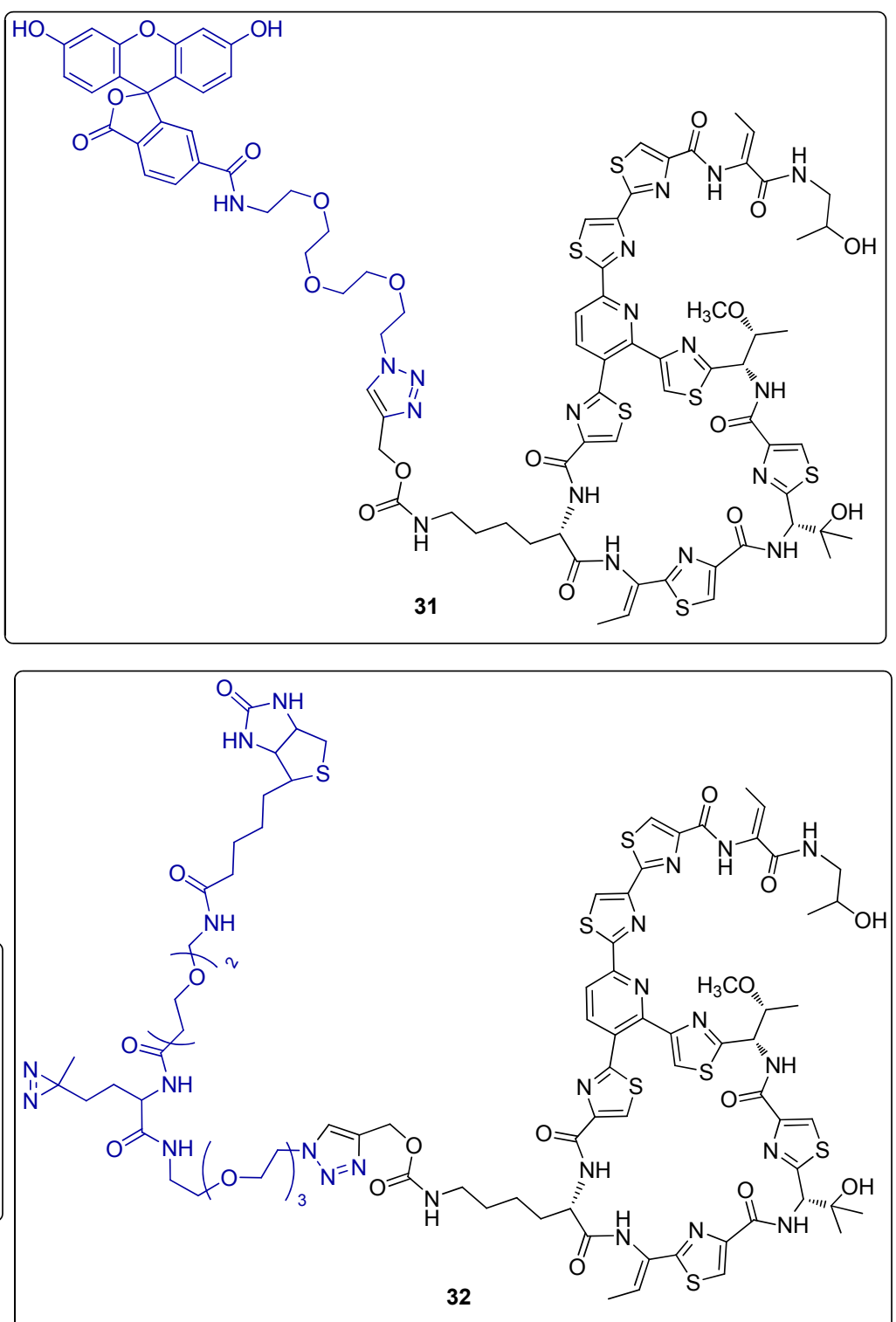

图 5 非天然氨基酸引入 thiocillin 中获得其类似物

Figure 5 Production of thiocillin analogues by incorporation of noncanonical amino acids 
入的非天然氨基酸 ProcK 的末端连有炔基, 因此可以通 过 Click 反应引入其他基团. Click 反应特异性地发生在 炔基上，因此不会与结构中其他官能团发生副反应，这 一点优于化学半合成中通过 Michael 加成(因为硫肽结 构中一般包含有多个双键)引入其他基团. 他们通过 Click 反应引入苂光基团获得了化合物 31. 更有意义的 是, 带有生物素的活性探针 32 为证明 thiocillin 和 L11 蛋白之间的直接相互作用提供了有力的证据. 因此, 硫 肽中引入非天然氨基酸不仅能够获得多种硫肽类似物, 探针的引入还能为研究人类微生物群落中微生物-微生 物间以及微生物一宿主间相互作用、未知的抗肿瘤靶点 提供可行的工具.

\section{2 基因缺失获取类似物}

由于生物合成途径的连续性可能会导致被敲除基 因之后的后修饰酶因为底物不识别而不能得到预期产 物, 因此, 只能敲除分子成熟过程中较靠后的后修饰基 因才有可能获得成熟的硫肽类似物. 2011 年, 刘文小 组 ${ }^{[35]}$ 在 thiostrepton A 体系中敲除 TsrB(酯水解酶) 后获得 了末端甲酯化 thiostrepton $\mathrm{A}$ 类似物, 敲除 $\mathrm{TsrC}$ (末端酰 胺化酶)后获得末端为羧酸的 thiostrepton $\mathrm{A}$ 类似物(图 6). 抗菌活性及水溶性测试表明: 甲酯化类似物活性提 高了 8 倍, 但溶解度降低为 thiostrepton A 的 $1 / 16$; 羧酸 类似物活性降低为 thiostrepton $\mathrm{A}$ 的 $1 / 8$, 但溶解度提高 了 1.5 倍.

\section{3 前体肽的定点突变获取类似物}

在 thiocillin 体系中, 2010 年 Walsh 小组 ${ }^{[36}$ 将其大环 骨架上所有氨基酸进行了突变(图 7A). Thiocillin 结构中 14 个氨基酸分别突变为丙氨酸(Ala)的结果表明: 除了
Cys11 及与核心氮杂六元环形成相关的 Ser1 和 Ser10 的 突变没能获得成熟 thiocillin 类似物外, 其他氨基酸的突 变都能获得成熟的 thiocillin 类似物. 获得的这些 thiocillin 类似物中除了 Val6Ala, Thr8Ala, Thr13Ala 及 Thr14Ala 保留了 thiocillin 的生物活性外, 其余位点突变 获得的成熟化合物均不再具有生物活性. Thiocillin 骨架 上 Cys 分别突变为丝氨酸(Ser)的结果表明: Cys11 的突 变没能获得 thiocillin 类似物, Cys5 的突变获得了丝氨酸 没有发生修饰的 thiocillin 类似物, Cys2, Cys7, Cys9 及 Cys12 的突变获得了丝氨酸没有发生修饰及修饰为啞唑 (噁唑啉)共存的 thiocillin 类似物. 获得的这些 thiocillin 类似物都不再具有抗菌活性. Thr3, Thr4, Val6, Thr8 位点 突变成丙氨酸(Ala)外的其他氨基酸结果表明: 虽然这 些位点的突变能够获得 thiocillin 类似物, 但它们均不再 具有抗菌活性. 2012 年, Bowers 等 ${ }^{[37]}$ 还采取了两种不同 的策略来获得不同环大小的 thiocillin 类似物: 一种是通 过删除 Thr3 (3 位苏氨酸)或在 Thr3 和 Thr4 之间引入 1 个到 3 个额外的甘氨酸获得了环大小为 $23,29,32$ 和 35 个原子的 thiocillin 类似物. 另外一种策略是在体内对六 元环形成的区域通过置换或者插入的方式引入丝氨酸 以获得不同环大小的类似物. 生物活性测试表明, 通过 这两种策略获得的 thiocillin 类似物都不再具有抗菌活 性. 根据 thiocillin 中氨基酸突变的结果可以得出其构效 关系的规律: Cys11 及与核心氮杂六元环形成相关的 Ser1 和 Ser10 的突变不能获得成熟的化合物; 大环结构 上 Cys2, Thr3, Thr4, Cys5, Cys7 及 Cys9 的突变虽然能获 得成熟的化合物，但均不再具有抗菌活性; 大环结构上 Val6, Thr8 及末端尾巴 Cys12, Thr13Ala, Thr14Ala 的突 变能获得抗菌活性保持的 thiocillin 类似物.
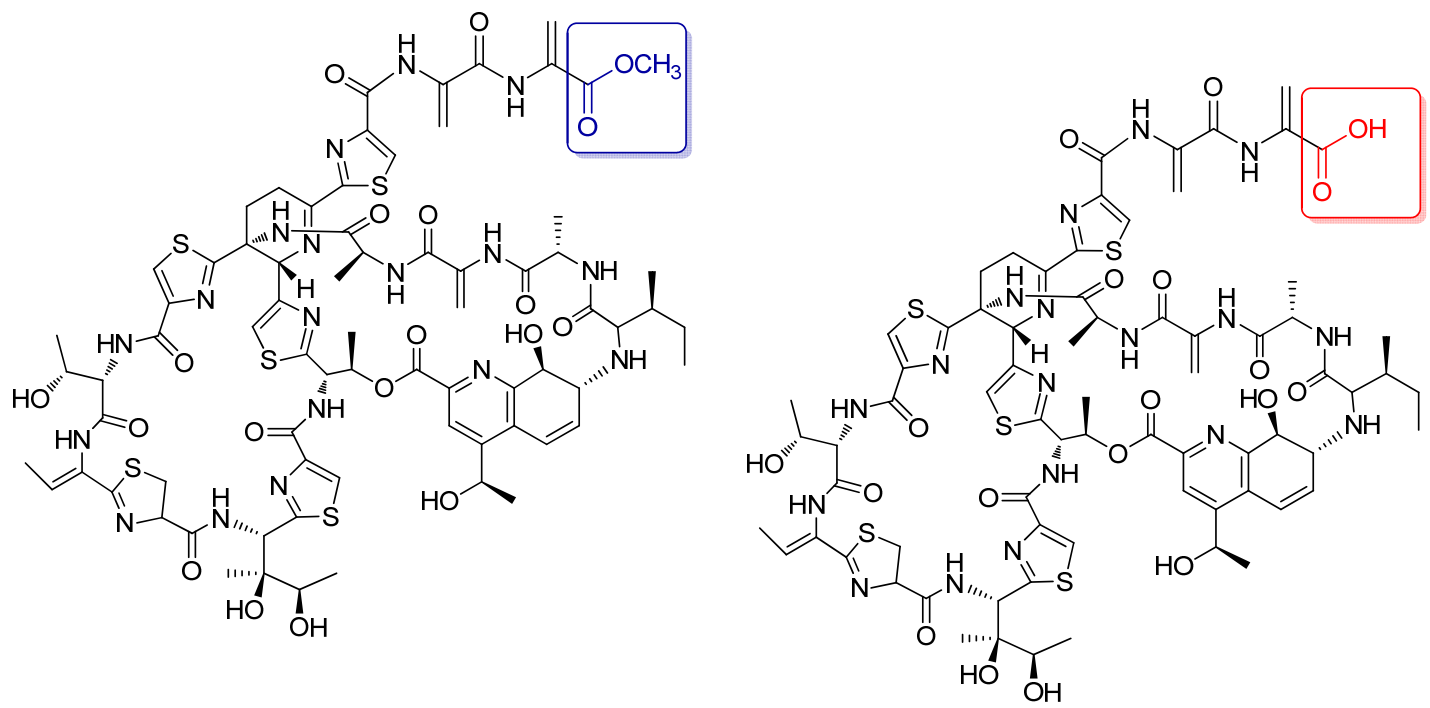

图 6 基因缺失获得 thiostrepton $\mathrm{A}$ 类似物

Figure 6 Production of thiostrepton A analogues by gene deletion 

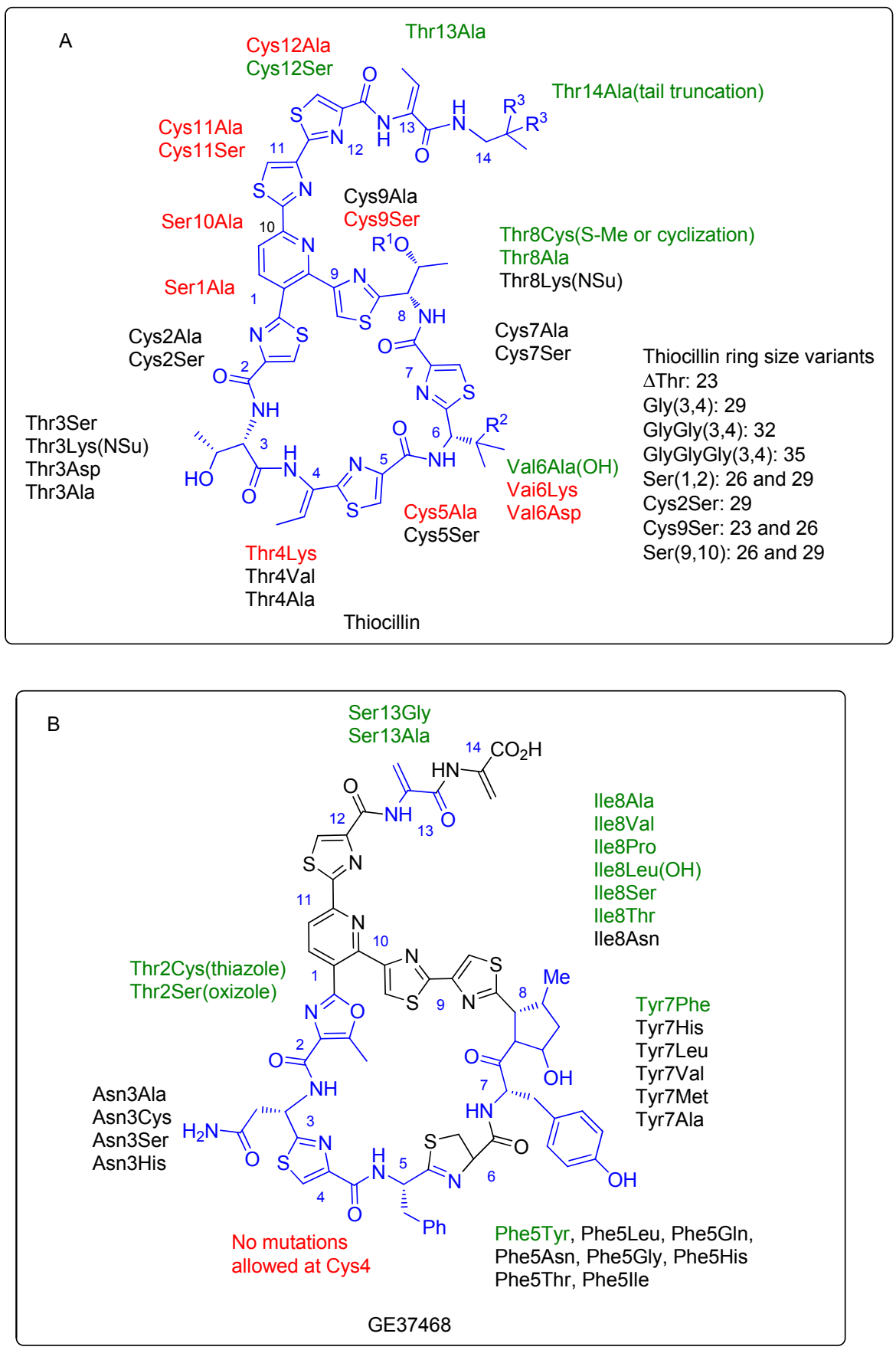

图 7 前体肽突变获得的 thiocillin 和 GE37468 的类似物

Figure 7 Production of thiocillin and GE37468 analogues by pre-peptide mutagenesis

在 GE37468 体系(图 7B)中, 2012 年 Walsh 小组 ${ }^{[38]}$ 通过密码子随机突变的方法对 GE37468 中 7 个位点 (Thr2, Asn3, Cys4, Phe5, Tyr7, Ile8, Ser13)进行点突变共 获得了 133 种突变株, 并利用高通量 MALDI-TOF 检测 手段对 133 种突变株的发酵产物进行了检测. 结果表明: 将 $\mathrm{Thr} 2$ 分别突变成半胱氨酸(Cys)或丝氨酸(Ser)后得到 的产物获得了 2 位形成噻唑或噁唑的 GE37468 类似物, 并且抗菌活性得到了保持. Phe5 只有突变成与其性质相 近的酪氨酸(Tyr)后才能获得抗菌活性保持的产物, 突变
成其他氨基酸获得的是抗菌活性丧失的产物. 类似地, Tyr7 只有突变成与其性质相近的 Phe 才能获得活性保持 的产物. Ile8 的突变获得的成熟分子中除 Ile8Asn 不再具 有抗菌活性外, 其余 6 种成熟分子的抗菌活性都得到了 保持. Cys4 的突变未能获得成熟的分子. Asn 3 的突变获 得的 GE37468 类似物均不再具有抗菌活性, 预示着 3 位 天门冬氨酸是 GE37468 发挥抗菌活性的关键位点. 在 29 环硫肽类抗生素 GE2270A, GE37468, baringolin 及 thiomuracin 的 3 位也都是保守的天门冬氨酸, 这表明 3 
位天门冬氨酸在 29 环硫肽类抗生素发挥抗菌活性上起 着重要的作用. 总之, 它们从 133 种突变株中共检测到 29 种成熟的 GE37468 类似物, 其中 12 种类似物的抗菌 活性得到保持, 1 种类似物(Thr2 Cys(thiozole))的抗菌活 性有所提高.

在 thiostrepton A 体系中, 2014 年刘文小组 ${ }^{[39]}$ 通过对 1 位氨基酸的突变及 2 位氨基酸的突变获得了抗菌活性 提高的 Ile1Val 和抗菌活性略有降低的 Ala2Dha. 进一 步, 他们对 thiostrepton A 结构肽 1 位的异亮氨酸进行了 饱和点突变. 点突变结果表明: 1 位异亮氨酸可以突变 为非极性氨基酸(Phe, Leu, Met, Ala, Trp, Pro)并产生相 应的 thiostrepton A 类似物. 相反, 当 1 位异亮氨酸突变 成极性氨基酸中的中性氨基酸(Ser, Thr, Gly, Tyr, Cys, Gln, Asn)、酸性氨基酸(Asp, Glu) 以及碱性氨基酸(Lys, Arg, His)后，突变株不再产生硫肽分子. 在此基础上, 他们通过双突变(结构肽 1 位异亮氨酸突变成缬氨酸, 结构肽 2 位丙氨酸突变成丝氨酸) 以期实现盐屋霉素 (siomycin) 在 thiostrepton A 宿主菌中的产生. Siomycin 作 为 thiostrepton $\mathrm{A}$ 的天然类似物比 thiostrepton $\mathrm{A}$ 具有更 好的药用生物活性. 有意思的是, 双突变突变株 SL-Ile1Ala-Val2Ser 不仅产生了预期的 siomycin，还产生 了 2 位丝氨酸未发生脱水的新型硫肽类抗生素 $\mathrm{OH}-$ siomycin (OH-SIO)(图 8). 水溶性和抗菌活性测试表明: TSR-Ile1Met, TSR-Ile1 Ala 和 OH-SIO 的水溶性有了很大 的提高, 其中 $\mathrm{OH}-\mathrm{SIO}$ 的水溶性相比于 thiostrepton A 提 高了近一倍. 除 siomycin 外, 其余 5 种 thiostrepton $\mathrm{A}$ 类 似物对测试的耐药菌的抗菌活性都有了明显降低，这表 明 thiostrepton A 结构肽 1 位氨基酸残基在其抗菌活性中 扮演未知的重要角色 ${ }^{[40]}$.

2015 年, Zhang 小组 ${ }^{[4]}$ 在 Ala4 的饱和点突变实验中 发现, 除 Ala4 突变为 Arg, Asp, Glu, Lys 和 Pro 没有得到 成熟的化合物外, 突变成其余 14 种天然氨基酸共获得 了 16 种成熟化合物(图 9). 其中 Ala4 突变为半胱氨酸 (Cys)后, 获得了 4 位半胱氨酸上颈基与 17 位脱氢丙氨 酸加成的产物 Ala4Cys F1 和 Ala4Cys F2. Ala4 突变为丝 氨酸(Ser)后获得了丝氨酸没有发生修饰及发生脱水修 饰共存的化合物, 而 Ala4 突变为苏氨酸(Thr)后仅获得 苏氨酸发生脱水修饰的化合物. 生物活性测试发现, 16 种化合物除 Ala4Cys F1 和 Ala4Cys F2 的抗菌活性丧失 外, 其余 14 种化合物的抗菌活性都得到了一定程度上 的保持; 16 种化合物的体外抑制蛋白翻译活性却相当. 2016 年, Wendy 小组在对 Ala2 的饱和点突变中发现 ${ }^{[42]}$ : 将 Ala2 突变成 Gly, Met, Phe, Tyr, Ser, Thr, Ile 和 Val 能 获得 8 种成熟的化合物. 其中 Ala2 突变成丝氨酸(Ser) 和苏氨酸(Thr)后都是获得发生脱水修饰的化合物. 将
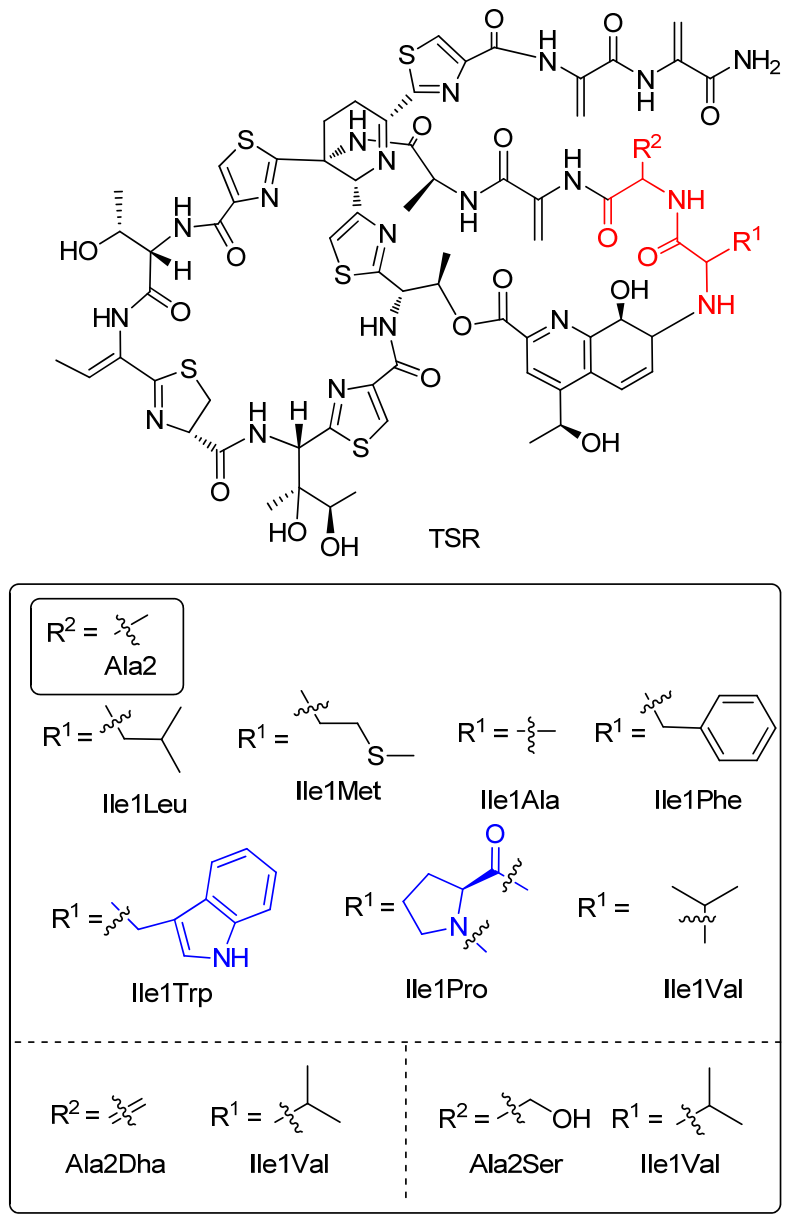

图 8 前体肽突变获得 thiostrepton A 的类似物(刘文小组) Figure 8 Production of thiostrepton A analogues by pre-peptide mutagenesis (Liu et al.)

Ala2 突变成异亮氨酸(Ile)或顺氨酸(Val)后, 得到的是 1 位异亮氨酸缺失的缩环产物 Ala2Ile- $\Delta$ Ile1 和 Ala2Val$\Delta$ Ile1. 生物活性测试发现: 8 种化合物除 Ala2Ile- $\Delta$ Ile1 和 Ala2Val- $\Delta$ Ile1 的抗菌活性丧失外，其余 6 种化合物的 抗菌活性都得到了一定程度上的保持; 体外抑制蛋白翻 译活性 Ala2Ile- $\Delta$ Ile1 和 Ala2Val- $\Delta$ Ile1 明显降低而其余 6 种化合物得到保持.

\section{3 前体导向的生物合成和突变生物合成}

传统的前体导向生物合成指的是在培养基中加入 前体小分子并利用野生菌株来产生次级代谢产物，该方 法得到的大多是次级代谢产物及其结构类似物的多组 分混合物. 前体导向的突变生物合成首先构建前体生物 合成途径被阻断的突变菌株，然后在培养基中加入前体 小分子或其类似物，利用突变菌株来产生次级代谢产 物，该方法得到的是次级代谢产物或其结构类似物的单 一组分. 

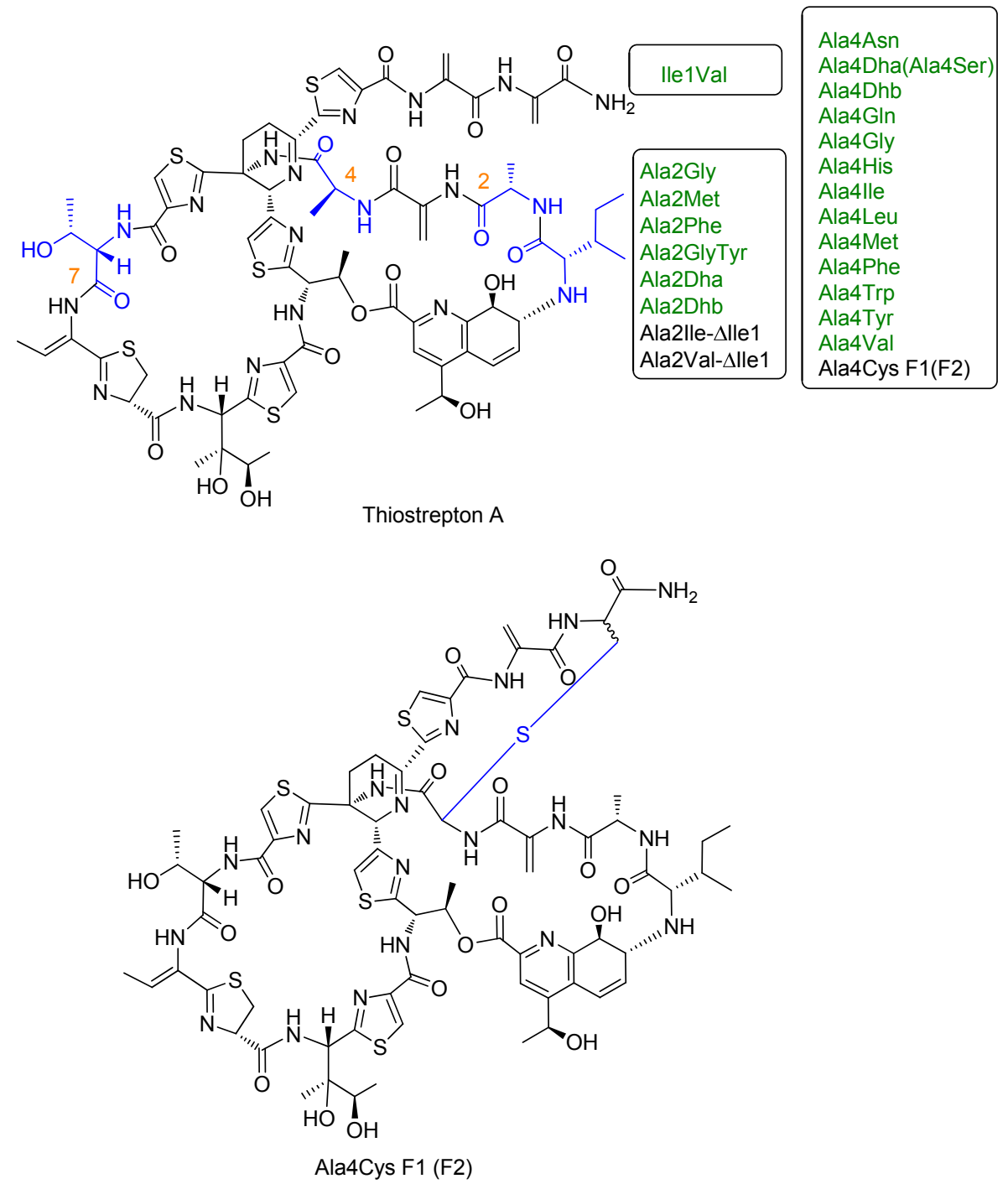

图 9 前体肽突变获得 thiostrepton A 的类似物(Wendy 小组)

Figure 9 Production of thiostrepton A analogues by pre-peptide mutagenesis (Wendy et al.)

很多双环硫肽的侧环中包含有吲哚酸(MIA)或者喹 萗啶酸(QA), 生物合成研究表明这两个模块是由色氨 酸经修饰后引入的, 他们独立于前体肽氨基酸序列之 外, 因此通过喂养色氨酸类似物来获取硫肽类似物是可 行的 ${ }^{[43,44]}$. 在 nosiheptide 的类似物合成中, 2011 年, 刘 文小组 ${ }^{[45]}$ 将 5 -F-色氨酸喂养到 nosiheptide 产生菌 $S$. actuosus 中, 利用前体导向的生物合成方法获得了氟代 的 nosiheptide(图 10,A). 在 thiostrepton A 的类似物合成 中, 2015 年, 刘文小组 ${ }^{[46]}$ 通过化学合成了 $5^{\prime}-F-Q A$, 6'-F-QA 及 12'-Me-QA, 并将这三种 QA 类似物喂养到 QA 生物合成阻断的 tsrT 突变株中, 利用前体导向的突 变生物合成方法获得了 5'-F-TSR, 6'-F-TSR 和 12'-Me-TSR(图 10, B). 对这三个 thiostrepton A 类似物抗 菌活性测试发现, 三者对多种革兰氏阳性菌的抗菌活性 都优于 thiostrepton A. 这表明侧环上 QA 结构的微调(取
代基的引入)不会影响其整体抗菌活性并且还有可能获 得抗菌活性提高的 thiostrepton A 类似物. 他们在研究这 三种 thiostrepton A 类似物对胞内菌 M. marinum 的抑制 机制时发现: 它们能够分别对 M. marinum 及其宿主发 挥作用而表现为双功能作用模式(MOA). 即它们除了能 够通过直接与 M. marinum 的 $50 \mathrm{~S}$ 核糖体亚基结合(即 TSR 的抗菌机制)抑制 M. marinum 外, 还能够诱导 $M$. marinum 的宿主细胞发生由内质网应(ER-stress)介导的 自噬, 从而达到抑菌效果 ${ }^{[47]}$. 至此, thiostrepton $\mathrm{A}$ 是目 前发现的唯一一例主动对胞内菌及其宿主细胞同时发 挥作用以达到抗菌效果的抗生素. 这一发现为发展抑制 胞内菌感染的抗生素提供了一种新思路. 进一步, 2016 年, 刘文小组 ${ }^{[88]}$ 通过将 5'-F-QA, 6'-F-QA 及 12'-Me-QA 喂养到 tsrB 突变株中, 获得了 5'-F-cmTSR, 6'-F-cmTSR 和 12'-Me-cmTSR, 这些类似物的抗菌活性较 thiostrepton 


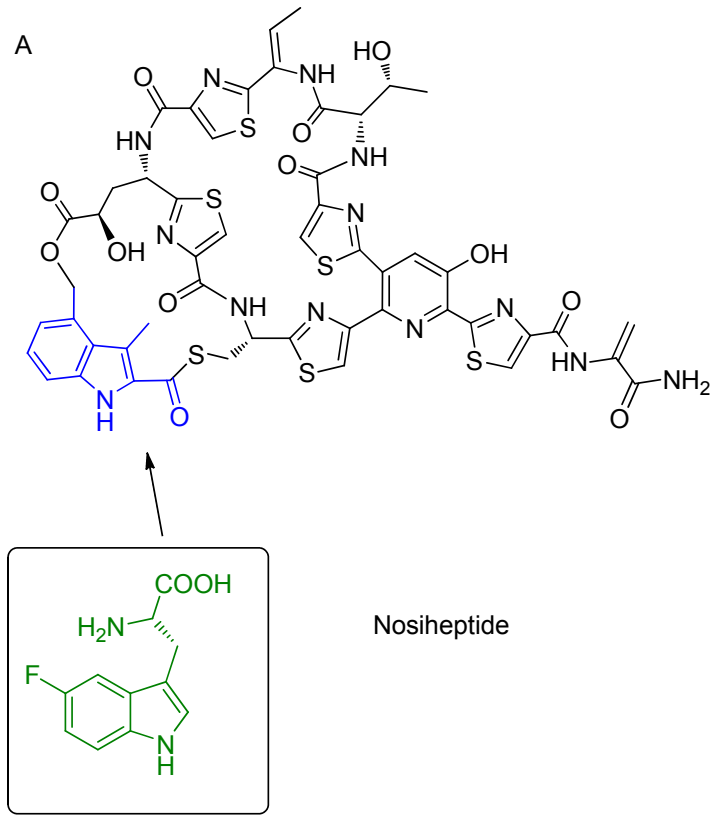

C

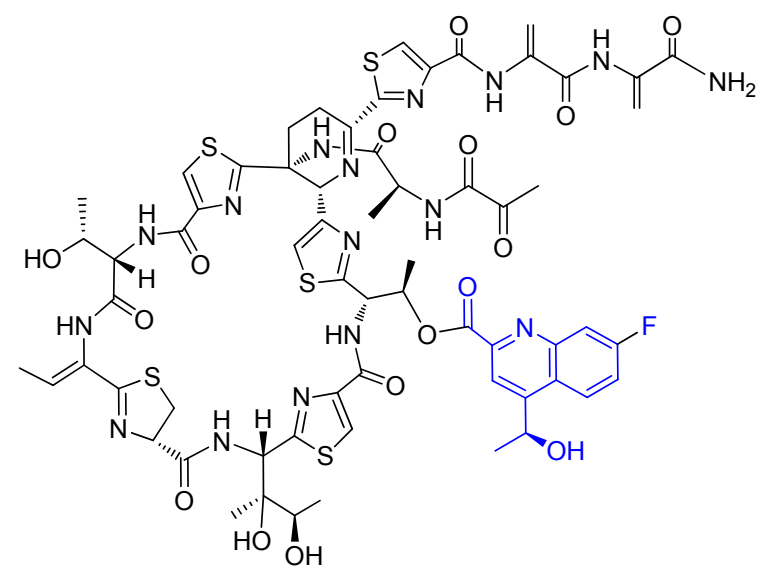

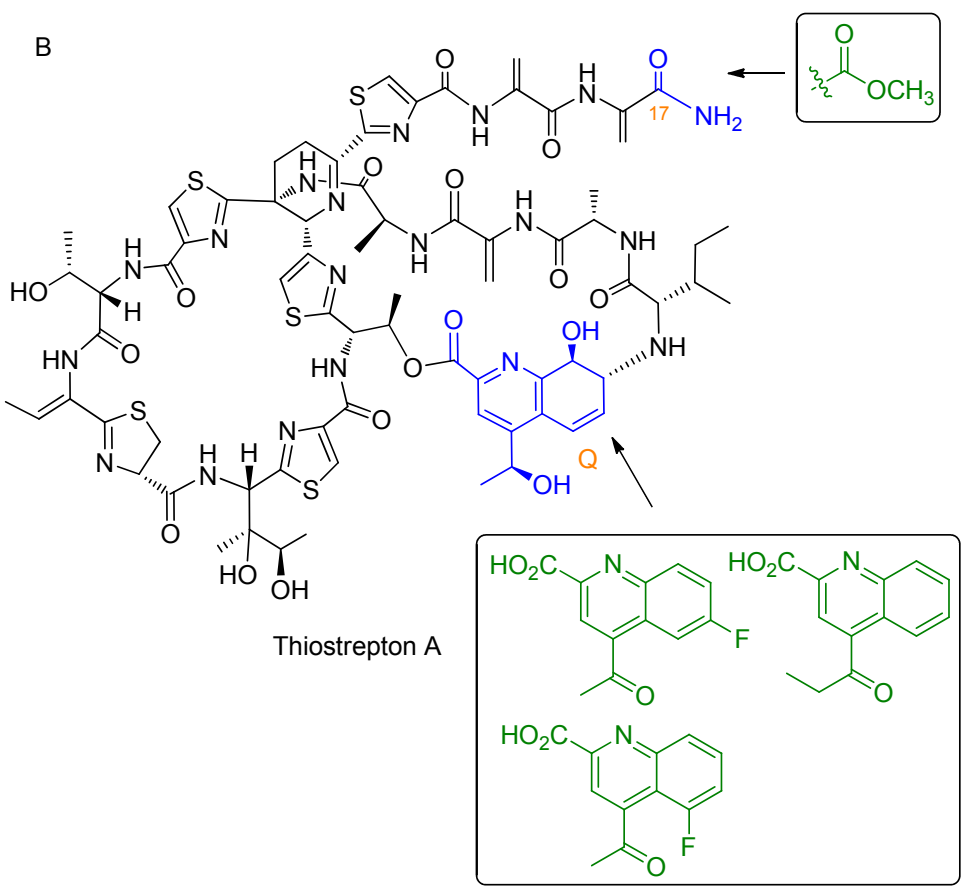

D

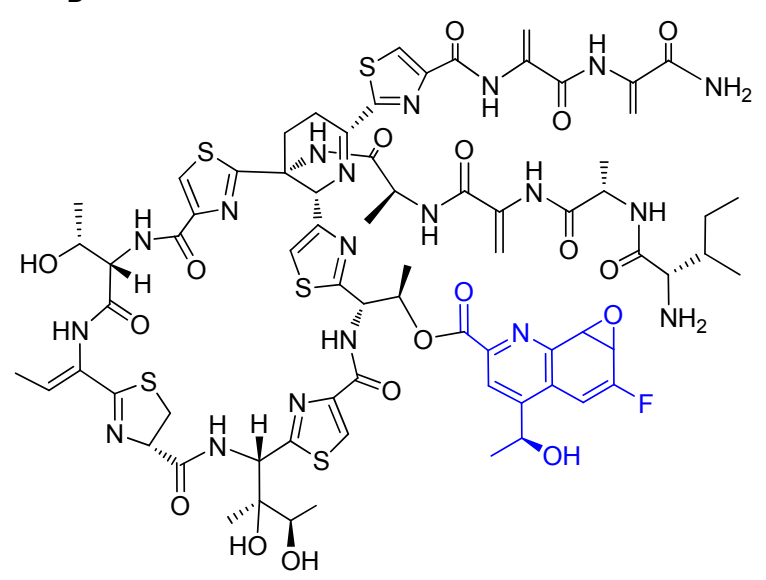

图 10 前体导向的生物合成和突变生物合成获得的硫肽类似物

Figure 10 Production of analogues by precursor-directed biosynthesis and mutational biosynthesis

$\mathrm{A}$ 提高 10 倍以上, 而且对于艰难梭状芽(Clostridium difficile)的抗菌效果较万古霉素提高 50 倍以上. 在随后 的研究过程中，他们基于同样的策略使用不同的合成前 体对相同的突变菌株进行化学喂养, 却发现了与预期产 物不一致的硫肽类似物. 在使用前体 7-F-QA 喂养突变 株 $\Delta$ tsrT 时，它们发现了一个侧环结构不完整的氟代 thiostrepton A 类似物(图 10, C), 其中喹菜啶酸(QA)结构 单元没有发生相应的氧化后修饰. 以此种具备二酮结构 特征的 TSR 分流产物(shunt product)作为预期, 他们研 究了 TSR 生物合成途径与分子骨架氧化后修饰相关的 基因功能, 通过基因敲除/回补、化学喂养、体外酶学测 活等手段, 最终确定 P450 蛋白 TsrP 负责了 thiostrepton $\mathrm{A}$ 侧环形成过程中 $\mathrm{QA}$ 结构单元的环氧化反应 ${ }^{[49]}$. 在最
近的研究中，他们继续沿用前体导向突变生物合成的策 略，通过优化 6'-F-QA 作为前体喂养突变株 $\Delta \mathrm{tsrT}$ 后的 发酵时间，成功分离、鉴定了一个侧环尚未关闭但 QA 基团已经发生环氧化的关键氟代中间体(图 10, D). 正是 由于氟原子的引入，降低了催化侧环形成的关键蛋白的 酶活性，从而导致了这样一个中间体的短暂积累; 而继 续延长发酵时间，该中间体会被转化为最终成熟的 6'-F-TSR 分子. 该中间体的分离和鉴定为 thiostrepton A 侧环生物合成途径中的酶学机制研究奠定了重要基础. 通过生物信息学分析，他们锁定了 thiostrepton A 生物合 成基因簇中一个功能尚未得到归属的基因 tsrI, 其编码 的蛋白 TsrI 隶属于 $\alpha / \beta$ 水解酶超家族. 在此基础上综合 使用基因敲除/回补、化学半合成模拟底物制备、体外酶 
学测活、化学喂养等手段, 证实了 TsrI 是 thiostrepton A 侧环生物合成过程中的关键蛋白, 该蛋白同时负责了先 导肽的切除和 TSR 侧环关闭过程的大环化反应. 关于 TsrI 功能的阐明, 不但解决了长期以来困扰人们的双环 硫肽抗生素侧环如何关闭的问题, 更拓展了人们对于 $\alpha / \beta$ 水解酶这一超家族蛋白生化功能的进一步认识和理 解, 同时为基于合成生物学策略改造双大环硫肽类抗生 素分子结构的研究奠定了基础 ${ }^{[50]}$.

\section{4 总结与展望}

随着致病菌临床耐药性的日益严峻, 硫肽类抗生素 优良的生物活性以及独特的构效关系和作用机制重新 引起了化学家和生物学家的研究兴趣, 但是硫肽类分子 本身并不适于直接作为药物或者化学探针. 近年来化学 家们通过化学半合成等手段获得了一些生物活性或水 溶性有所提高的硫肽衍生物(如 LEF571 和 NAI003), 但 是由于受反应条件及可修饰位点的限制, 目前人们对硫 肽类抗生素的化学半合成仍然只是集中在其 $\mathrm{C}$ 端尾部. 与其他常见的临床抗生素相比, 通过硫肽类抗生素 “模 板化”的生物合成途径对其进行分子改造显得尤为简洁, 即通过对于其前体肽序列的定点突变即可获得一系列 的结构衍生物. 因此, 在了解了硫肽抗生素生物合成途 径中的化学逻辑和酶学机制后, 有助于人们通过基于合 成生物学的策略进一步拓展硫肽分子结构和功能的多 样性.

本文对于目前常用的制备硫肽类抗生素衍生物的 方法进行了归纳和总结, 从化学和生物学两方面进行了 比较. 我们认为, 在未来的研究中, 通过“化学辅助的合 成生物学” 手段能够进一步构建成员更多的以硫肽为代 表的复杂天然产物文库, 即通过合成生物学构筑具有某 种特定生物活性的复杂分子骨架, 通过化学半合成或使 用合成的前体喂养相应突变株, 从而进行骨架侧链的修 饰以及生物活性的 “微调”. 我们相信, 在不久的将来 基于硫肽类抗生素分子骨架的新型药物和化学探针将 会在临床治疗和基础研究中大展神威.

\section{Referenes}

[1] Bagley, M. C.; Dale, J. W.; Merritt, E. A.; Xiong, X. Chem. Rev. 2005, 105, 685 .

[2] Li, C.; Kelly, W. L. Nat. Prod. Rep. 2010, 27, 153.

[3] Hensens, O. D.; Albers-Schönberg, G. Tetrahedron Lett. 1978, 19, 3649 .

[4] Naidu, B. N.; Sorenson, M. E.; Zhang, Y.; Kim, O. K.; Matiskella, J. D.; Wichtowski, J. A.; Connolly, T. P.; Li, W.; Lam, K. S.; Bronson, J. J.; Pucci, M. J.; Clark, J. M.; Ueda, Y. Bioorg. Med. Chem. Lett. 2004, 14, 5573.

[5] Zhang, C.; Herath, K.; Jayasuriya, H.; Ondeyka, J. G.; Zink, D. L.; Occi, J.; Birdsall, G.; Venugopal, J.; Ushio, M.; Burgess, B.; Masurekar, P.; Barrett, J. F.; Singh, S. B. J. Nat. Prod. 2009, 72, 841.
[6] Zhang, C.; Occi, J.; Masurekar, P.; Barrett, J. F.; Zink, D. L.; Smith, S.; Onishi, R.; Ha, S.; Salazar, O.; Genilloud, O.; Basilio, A.; Vicente, F.; Gill, C.; Hickey, E. J.; Dorso, K.; Motyl. M.; Singh, S. B. J. Am. Chem. Soc. 2008, 130, 12102.

[7] Bower, J.; Drysdale, M.; Hebdon, R.; Jordan, A.; Lentzen, G.; Matassova, N.; Murchie, A.; Powles, J.; Roughley, S. Bioorg. Med. Chem. Lett. 2003, 13, 2455.

[8] Naidu, B. N.; Sorenson, M. E.; Bronson, J. J.; Pucci, M. J.; Clark, J. M.; Ueda, Y. Bioorg. Med. Chem. Lett. 2005, 15, 2069.

[9] Naidu, B. N.; Sorenson, M. E.; Matiskella, J. D.; Li, W.; Sausker, J. B.; Zhang, Y.; Connolly, T. P.; Lam, K. S.; Bronson, J. J.; Pucci, M. J.; Yang, H.; Ueda, Y. Bioorg. Med. Chem. Lett. 2006, 16, 3545.

[10] Regueiro-Ren, A.; Ueda, Y. J. Org. Chem. 2002, 67, 8699.

[11] Connolly, T. P.; Regueiro-Ren, A.; Leet, J. E.; Springer, D. M.; Goodrich, J.; Huang, X.; Pucci, M. J.; Clark, J. M.; Bronson, J. J.; Ueda, Y. J. Nat. Prod. 2005, 68, 550.

[12] Xu, L.; Farthing, A. K.; Shi, Y.; Meinke, P. T.; Liu, K. J. Org. Chem. 2007, 72, 7447.

[13] Xu, L.; Farthing, A. K.; Dropinski, J. F.; Meinke, P. T.; McCallum, C.; Leavitt, P. S.; Hickey, E. J.; Colwell, L.; Barrett, J.; Liu, K. Bioorg. Med. Chem. Lett. 2009, 19, 3531.

[14] Hrnciar, P.; Ueda, Y.; Huang, S.; Leet, J. E.; Bronson, J. J. J. Org. Chem. 2002, 67, 8789.

[15] Pucci, M. J.; Bronson, J. J.; Barrett, J. F.; DenBleyker, K. L.; Discotto, L. F.; Fung-Tomc, J. C.; Ueda, Y. Antimicrob. Agents Chemother. 2004, 48, 3697.

[16] Regueiro-Ren, A.; Naidu, B. N.; Zheng, X.; Hudyma, T. W.; Connolly, T. P.; Matiskella, J. D.; Zhang, Y.; Kim, O. K.; Sorenson, M. E.; Pucci, M. J.; Clark, J.; Bronson, J. J.; Ueda, Y. Bioorg. Med. Chem. Lett. 2004, 14, 171.

[17] Naidu, B. N.; Sorenson, M. E.; Hudyma, T.; Zheng, X.; Zhang, Y.; Bronson, J. J.; Pucci, M. J.; Clark, J. M.; Ueda, Y. Bioorg. Med. Chem. Lett. 2004, 14, 3743.

[18] Golik, J.; Wong, H. S. L.; Chen, S. H.; Doyle, T. W.; Wright, J. J. K.; Knipe, J.; Rose, W. C.; Casazzam, A. M.; Vyas, D. M. Bioorg. Med. Chem. Lett. 1996, 6, 1837.

[19] Tavecchia, P.; Gentili, P.; Kurz, M.; Sottani, C.; Bonfichi, R.; Selva, E.; Lociuro, S.; Restelli, E.; Ciabatti, R. Tetrahedron 1995, 51, 4867.

[20] Tavecchia, P.; Kruz, M.; Colombo, L.; Bonfichi, R.; Selva, E.; Lociuro, S.; Marzorati, E.; Ciabatti, R. Tetrahedron 1996, 52, 8763.

[21] Lociuro, S.; Tavecchia, P.; Marzorati, E.; Goldstein, B. P.; Denaro, M.; Ciabatti, R. J. Antibiot. 1997, 50, 344..

[22] LaMarche, M. J.; Leeds, J. A.; Amaral, K.; Brewer, J. T.; Bushell, S. M.; Dewhurst, J. M.; Dzink-Fox, J.; Gangl, E.; Goldovitz, J.; Jain, A.; Mullin, S.; Neckermann, G.; Osborne, C.; Palestrant, D.; Patane, M. A.; Rann, E. M.; Sachdeva, M.; Shao, J.; Tiamfook, S.; Whitehead, L.; Yu, D. J. Med. Chem. 2011, 54, 8099.

[23] LaMarche, M. J.; Leeds, J. A.; Dzink-Fox, J.; Mullin, S.; Patane, M. A.; Rann, E. M.; Tiamfook, S. Bioorg. Med. Chem. Lett. 2011, 21, 3210 .

[24] LaMarche, M. J.; Leeds, J. A.; Amaral, A.; Brewer, J. T.; Bushell, S. M.; Deng, G.; Dewhurst, J. M.; Ding, J.; Dzink-Fox, J.; Gamber, G.; Jain, A.; Lee, K.; Lee, L.; Lister, T.; McKenney, D.; Mullin, S.; Osborne, C.; Palestrant, D.; Patane, M. A.; Rann, E. M.; Sachdeva, M.; Shao, J.; Tiamfook, S.; Trzasko, A.; Whitehead, L.; Yifru, A.; Yu, D.; Yan, W.; Zhu, Q. J. Med. Chem. 2012, 55, 2376.

[25] LaMarche, M. J.; Leeds, J. A.; Dzink-Fox, J.; Gunderson, K.; Krastel, P.; Memmert, K.; Patane, M. A.; Rann, E. M.; Schmitt, E.; Tiamfook, S.; Wang, B. J. Med. Chem. 2011, 54, 2517.

[26] Trzasko, A.; Leeds, J. A.; Praestgaard, J.; Lamarche, M. J.; McKenney, D. Antimicrob. Agents Chemother. 2012, 56, 4459.

[27] Ting, L. S.; Praestgaard, J.; Grunenberg, N.; Yang, J. C.; Leeds, J. A.; Pertel, P. Antimicrob. Agents Chemother. 2012, 56, 5946.

[28] Fabbretti, A.; He, C. G.; Gaspari, E.; Maffioli, S.; Brandi, L.; Spurio, R.; Sosio, M.; Jabes, D.; Donadio, S. Antimicrob. Agents Chemother. 2015, 59, 4560.

[29] Schoof, S.; Baumann, S.; Ellinger, B.; Arndt, H. D. ChemBioChem 
2009, 10, 242

[30] Baumann, S.; Schoof, S.; Bolten, M.; Haering, C.; Takagi, M.; Shin-ya, K.; Arndt, H. D. J. Am. Chem. Soc. 2010, 132, 6973.

[31] Myers, C. L.; Hang, P. C.; Ng, G.; Yuen, J.; Honek, J. F. Bioorg. Med. Chem. 2010, 18, 4231

[32] Jonker, H. R.; Baumann, S.; Wolf, A.; Schoof, S.; Hiller, F.; Schulte, K. W.; Kirschner, K. N.; Schwalbe, H.; Arndt, H. D. Angew. Chem., Int. Ed. Engl. 2011, 50, 3308.

[33] Hegde, N. S.; Sanders, D. A.; Rodriguez, R.; Balasubramanian, S. Nat. Chem. 2011, 3, 725.

[34] Luo, X.; Zambaldo, C.; Liu, T.; Zhang, Y.; Xuan, W.; Wang, C.; Reed, S. A.; Yang, P. Y.; Wang, R. E.; Javahishvili, T.; Schultz, P. G.; Young, T. S. Proc. Natl. Acad. Sci. U. S. A. 2016, 113, 3615.

[35] Liao, R.; Liu, W. J. Am. Chem. Soc. 2011, 133, 2852.

[36] Bowers, A. A.; Acker, M. G.; Koglin, A.; Walsh, C. T. J. Am. Chem. Soc. 2010, 132, 7519.

[37] Bowers, A. A.; Acker, M. G.; Young, T. S.; Walsh, C. T. J. Am. Chem. Soc. 2012, 134, 10313.

[38] Young, T. S.; Dorrestein, P. C.; Walsh, C. T. Chem. Biol. 2012, 19, 1600 .

[39] Guo, H.; Wang, J.; Li, Y.; Yu, Y.; Zheng, Q.; Wu, J.; Liu, W. Chem.
Sci. 2014, 5, 240.

[40] Duan, P.; Zheng, Q.; Lin, Z.; Wang, S.; Chen, D.; Liu, W. Org. Chem. Front. 2016, 3, 1254.

[41] Zhang, F.; Kelly, W. L. ACS Chem. Biol. 2015, 10, 998.

[42] Zhang, F.; Li, C.; Kelly, W. L. ACS Chem. Biol. 2016, 11, 415

[43] Zhang, Q.; Chen, D.; Lin, J.; Liao, R.; Tong, W.; Xu, Z.; Liu, W. J. Biol. Chem. 2011, 286, 21287.

[44] Duan, L.; Wang, S.; Liao, R.; Liu, W. Chem. Biol. 2012, 19, 443.

[45] Zhang, Q.; Li, Y.; Chen, D.; Yu, Y.; Duan, L.; Shen, B.; Liu, W. Nat. Chem. Biol. 2011, 7, 154

[46] Wang, S.; Zheng, Q.; Wang, J.; Zhao, Z.; Li, Q.; Yu, Y.; Wang, R.; Liu, W. Org. Chem. Front. 2015, 2, 106.

[47] Zheng, Q.; Wang, Q.; Wang, S.; Wu, J.; Gao, Q.; Liu, W. Chem. Biol. 2015, 22, 1002.

[48] Wang, S.; Zheng, Q.; Wang, J.; Chen, D.; Yu, Y.; Liu, W. Org. Chem. Front. 2016, 3, 496.

[49] Zheng, Q.; Wang, S.; Liao, R.; Liu, W. ACS Chem. Biol. 2016, 11, 2673.

[50] Zheng, Q.; Wang, S.; Duan, P.; Liao, R.; Chen, D.; Liu, W. Proc. Natl. Acad. Sci. U. S. A. 2016, 113, 14318.

(Zhao, C.) 